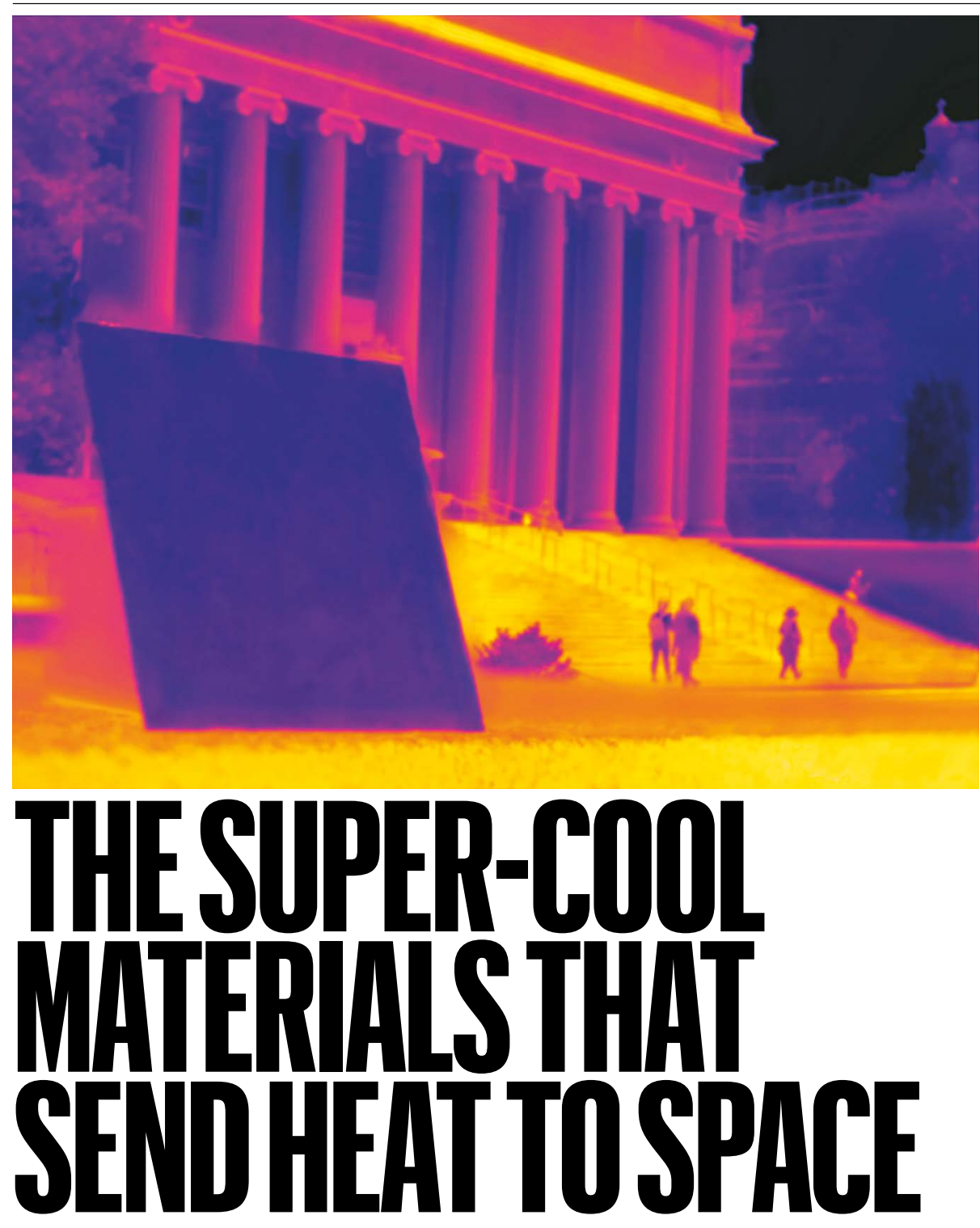

Paints, plastics and even wood can be engineered to stay cool in direct sunlight - but their role in displacing power-hungry air conditioners remains unclear. By XiaoZhi Lim

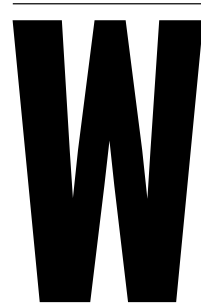

hen businessman Howard Bisla
was tasked with saving a local
shop from financial ruin, one
of his first concerns was energy
efficiency. In June 2018, he
approached his local electric-
ity provider in Sacramento,
California, about upgrading the lights. The provider had another idea. It offered to install an experimental cooling system: panels that could stay colder than their surroundings, even under the blazing hot sun, without consuming energy.

The aluminium-backed panels now sit on the shop's roof, their mirrored surfaces coated with a thin cooling film and angled to the sky. They cool liquid in pipes underneath that run into the shop, and, together with new lights,
A thermal image of a panel with a

'super-cool' coating outside

Columbia University in New York City.

blanket-like atmosphere absorbs most of it and radiates it back. Infrared rays between 8 and 13 micrometres in wavelength, however, are not captured by the atmosphere and leave Earth, escaping into cold outer space. As far back as the1960s, scientists sought to harness this phenomenon for practical use. But passive radiative cooling is noticeable only at night: in the daytime, sunlight bathes us in much more heat energy than we can send into space.

The new materials reflect a broad spectrum of light, in much the same way as mirrors or white paint do. In the crucial 8-13- $\mu \mathrm{m}$ part of the infrared spectrum, however, they strongly absorb and then emit radiation. When the materials point at the sky, the infrared rays can pass straight through the atmosphere and into space. That effectively links the materials to an inexhaustible heat sink, into which they can keep dumping heat without it coming back. As a result, they can radiate away enough heat to consistently stay a few degrees cooler than surrounding air; research suggests that temperature differences could exceed $10^{\circ} \mathrm{C}$ in hot, dry places ${ }^{2,3}$. David Sailor, who leads the Urban Climate Research Center at Arizona State University in Tempe, has termed them super-cool materials.

These materials might not only save on electricity bills, say enthusiasts, but also reduce a surge in demand for power-hungry refrigeration and air conditioning as the world warms. "My belief is that in four to five years, daytime radiative cooling systems will be the number one technology for buildings," says Mattheos Santamouris at the University of New South Wales in Sydney, Australia, who himself is working to improve such materials. "It is the air conditioner of the future."

A few researchers have even suggested that the materials might be considered as part of a geoengineering strategy, to help Earth shed heat to counteract global rising temperatures. "Rather than try to block the incoming heat from the Sun, can we just make Earth emit more?" asks Jeremy Munday, a physicist at the University of California, Davis.

But many scientists are cautious about these ideas. So far, theoretical estimates of how much electrical power can be saved have been based on data from small samples tested over short times. There are also doubts about the materials' ability to work in a wide variety of climates and places. The cooling effect works best in dry climates and with clear skies; when it's cloudy or humid, water vapour traps the infrared radiation. And the super-cool materials might not last in all weathers or fit easily to all buildings.

Another unknown is whether consumers will embrace the idea. Even the simple measure of replacing worn-out roofs with reflective 
white ones to cool houses has not been widely adopted by homeowners, says Sailor. His modelling work, however, suggests that use of a super-cool paint might double the energy savings compared with a white roof. "It's a bit of a game-changer - potentially," he says.

\section{Overcoming the Sun}

In 2012, Raman - who was completing his $\mathrm{PhD}$ with Fan on materials for harvesting solar energy - stumbled on old studies about passive radiative cooling, an effect he'd not heard of. Realizing that no one had worked out how to use it under direct sunlight, he examined the optical properties a material would need to overcome the Sun's heat. It must reflect the solar spectrum in wavelengths from 200 nanometres to $2.5 \mu \mathrm{m}$ even more effectively than white paint, which is already up to $94 \%$ reflective. And it must absorb and emit as close as possible to $100 \%$ of the wavelengths in the crucial 8-13- $\mu \mathrm{m}$ range (see 'Keeping their cool').

All this could be done by engineering materials at the nanoscale, Raman and Fan thought. Creating structures smaller than the wavelengths of light that will pass through them should enhance the absorption and emission of some wavelengths and suppress that of others.

The group came up with the idea to etch patterns into surfaces ${ }^{4}$ and published it in 2013. Then the team submitted a proposal to the US Advanced Research Projects Agency-Energy (ARPA-E) for funding to make it.

"I immediately thought, 'Wow, I'd really like to see somebody actually do this,'” recalls Howard Branz, then a programme director at ARPA-E in Washington DC, and now a technology consultant in Boulder, Colorado. “There'd been a lot of night-time radiative-cooling work, but to do it under broad, full sunlight is quite startling."

Branz gave the researchers US $\$ 400,000$ and a year. With so little time, the Stanford team decided to simplify the design and try layering materials in more familiar ways. To create something highly reflective, the researchers alternated four thin layers of materials that refract light strongly (hafnium dioxide) and weakly (silicon dioxide, or glass), a commonly used motif in optical engineering that works because of how light waves interfere as they pass through different layers. They used the same principle to amplify infrared emissions, depositing three thicker layers of the same materials on top.

When they tested their material outdoors ${ }^{1}$ it stayed almost $5^{\circ} \mathrm{C}$ cooler than the ambient temperature, even under direct sunlight of around 850 watts per square metre. (On a bright, clear day at sea level, the intensity of sunlight directly overhead reaches around $1,000 \mathrm{Wm}^{-2}$ ).

After that success, ARPA-E funded other proposals for super-cool materials. Among these was an idea from Xiaobo Yin and Ronggui

\section{KEEPING THEIR COOL}

'Super-cool' materials stay colder than their surroundings even in direct sunlight, by emitting heat that can pass through the atmosphere and into space.

\section{Transparent atmosphere}

Earth's blanket-like atmosphere absorbs most infrared wavelengths but is transparent to those between 8 and 13 micrometres.

Radiation from the Sun

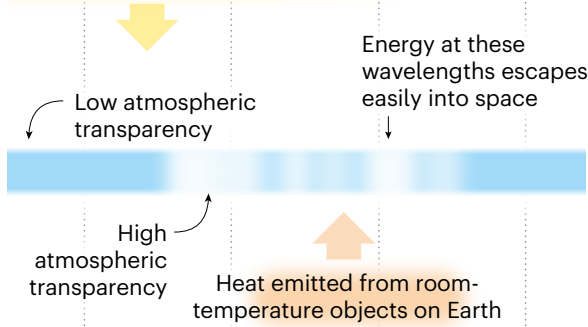

$\begin{array}{lc:cc}\begin{array}{l}\text { Ultraviolet, } \\ \text { X-rays }\end{array} & \begin{array}{c}\text { Visible } \\ \text { light }\end{array} & \begin{array}{c}\text { Infrared } \\ \text { (heat) }\end{array} & \begin{array}{c}\text { Radio } \\ \text { waves }\end{array} \\ \text { and } \mathrm{Y} \text {-rays } & \mathbf{1 1} & & \longrightarrow\end{array}$

$\leftarrow$

$$
\begin{array}{ccc}
0.1 & 10 \\
& \text { Wavelength }(\mu \mathrm{m})
\end{array}
$$$$
100
$$

Reflect and emit

Super-cool materials are extremely reflective (even more so than white paint), so they are relatively unaffected by sunlight. They also absorb wavelengths between 8 and $13 \mu \mathrm{m}$, then emit them into space.

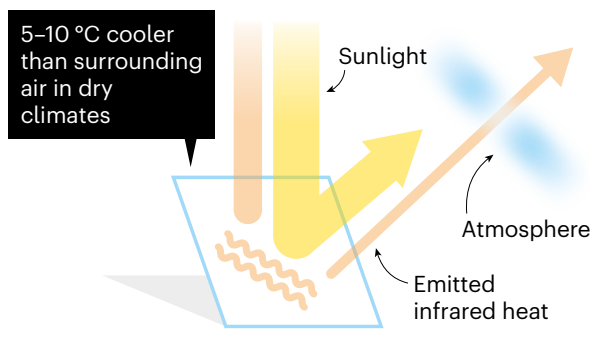

Yang at the University of Colorado Boulder, who wanted to make materials at large scale. They chose to work with cheap plastic and glass. Glass spheres of the right size - a few micrometres across - emit strongly in the $8-13-\mu \mathrm{m}$ range. Embedding these in a 50- $\mu \mathrm{m}$-thick film of transparent polymethylpentene - a plastic used in some lab equipment and cookware and backing this with reflective silver was sufficient to create a super-cool material ${ }^{5}$. More importantly, the researchers could make the film with roll-to-roll technology that churns out 5 metres per minute.

It turned out that many materials exhibit super-cooling if structured in the right way - not just exotic or speciality ones. In 2018, researchers at Columbia University in New York City and Argonne National Laboratory in Lemont, Illinois, reported a super-cool paint, based on a sprayable polymer coating ${ }^{6}$. Many polymers naturally emit in the infrared $8-13-\mu \mathrm{m}$ range because their chemical bonds, such as those between carbon atoms or between carbon and fluorine, eject packets of infrared light when they stretch and relax, explains team member Yuan Yang. The key was to strengthen the polymers' ability to reflect sunlight.

Yang's student Jyotirmoy Mandal - who is now a postdoctoral researcher in Raman's lab at the University of California, Los Angeles dissolved fluorinated polymer precursors in acetone with a small amount of water. This mixture can be sprayed onto a surface to create an even polymer coating with tiny water droplets dispersed through it. The volatile acetone dries first, followed by the water droplets, leaving behind pores that fill with air. The overall result is a white coating with pores inside that reflect the sunlight, Yang says.

Last May, the Colorado team reported another material: a cooling wood, created with Liangbing Hu and Tian Li at the University of Maryland, College Park. Just like polymers, wood contains chemical bonds that emit the right kind of infrared radiation, says Li. A net cooling effect can be achieved by chemically removing a rigid component called lignin to make the wood reflective and compressing the product to align its cellulose fibres and amplify infrared emissions ${ }^{7}$.

Scientists have also made super-cool thin films from polydimethylsiloxane (PDMS), a silicone material found in products such as lubricants, hair conditioners and Silly Putty, by spraying it onto a reflective backing. As recently as last August, Zongfu Yu at the University of Wisconsin-Madison and Qiaoqiang Gan at the State University of New York at Buffalo found that an aluminium film spray-coated with a $100-\mu$ m layer of PDMS stayed $11^{\circ} \mathrm{C}$ cooler than ambient air when placed in a campus car park in the middle of the day ${ }^{2}$.

\section{Staying cool}

Almost all the research teams have patented their inventions and are now trying to market them. Gan is working with industry partners, which he declined to name, to commercialize the PDMS-aluminium film. Columbia University has licensed its super-cool paint to New York start-up MetaRE, founded by Mandal and Yang's Columbia collaborator Nanfang $\mathrm{Yu}$, for development. MetaRE is also working with industry to develop the paint for roofing, refrigerated transportation, storage and textile applications, says chief executive April Tian. The product is "highly competitive" with conventional paints, she says.

Other start-ups have highlighted how much electricity their products could save. Fan and Raman have developed a proprietary system for SkyCool Systems' panels. In 2017, they predicted that the system could reduce the amount of electricity a building uses for cooling by $21 \%$ during the summer in hot, dry Las Vegas, Nevada ${ }^{8}$. Raman says the panels will pay for themselves in three to five years. Yin and Ronggui Yang have started a company in Boulder called Radi-Cool, to commercialize the glass-embedded plastic. Last January, they reported that the material could reduce 


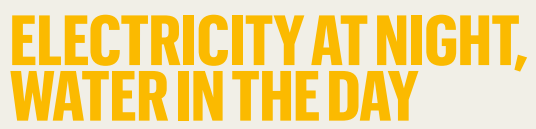

Super-cool materials have added benefits.

Materials that dump heat from Earth into space could have unexpected applications. They could, for instance, make it easier to harvest water from the atmosphere in the daytime. At night, water vapour condenses into dew on surfaces that lose heat to the clear night sky, an effect harnessed for centuries to capture water. Zongfu Yu at the University of Wisconsin-Madison and Qiaoqiang Gan at the State University of New York at Buffalo found that an aluminium film coated in polydimethylsiloxane could not only stay cool, but also enhance water condensation during the day ${ }^{12}$. The pair started a company in Buffalo called Sunny Clean Water to commercialize the device.

The temperature difference between a super-cool material and its surroundings could also be used to generate electricity at night - unlike solar panels, which work only in the day. Last September, Aaswath Raman, Shanhui Fan and Wei Li at Stanford University in California managed to produce a trickle of electricity - milliwatts per square metre - from such a nocturnal device ${ }^{13}$. That shows it's possible to make at least enough electricity at night to power a small LED. That's an exciting proof of concept, says Howard Branz, a technology consultant in Boulder, Colorado. But electricity from solar panels can be stored in batteries to generate much larger flows of electricity, so it's not yet clear whether the idea will be useful.

electricity consumption for cooling in the summer by $32-45 \%$ if it were integrated with water chillers in commercial buildings in Phoenix, Arizona; Miami, Florida; and Houston, Texas?. $\mathrm{Hu}$, meanwhile, has licensed the super-cool wood material to a Maryland-based firm he co-founded called InventWood. He predicts that it could save $20-35 \%$ of cooling energy across 16 US cities ${ }^{7}$.

But these estimates are based on experiments and models that are too limited to be extrapolated to whole buildings in cities, cautions Diana Ürge-Vorsatz, an environmental scientist at the Central European University in Budapest who specializes in climate-change mitigation. Actual energy savings and how quickly a super-cool material will pay for itself will depend on a building's structure, location and weather conditions, adds Yin.

Location is the biggest obstacle. "There are certain geographical regions where it just won't work because the atmosphere isn't dry

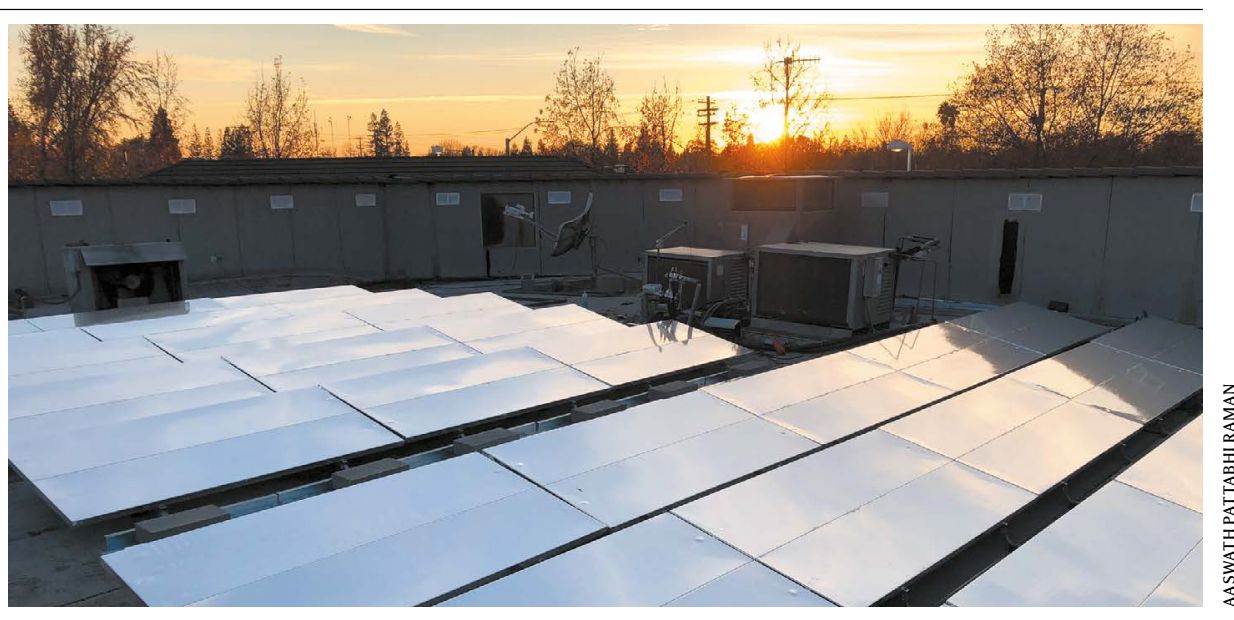

Super-cool panels on the roof of a shop in Sacramento, California.

enough," says James Klausner, a mechanical engineer at Michigan State University in East Lansing who served as an ARPA-E programme director after Branz and has funded some proposals in the field. But that's not too off-putting, he says, because the regions where the effect works well are arid areas such as the southwestern United States or the Middle East, which have high demands for air conditioning.

Another challenge is that radiative-cooling systems might increase heating costs in winter. To address this problem, Santamouris is trying to introduce a liquid layer on top of the super-cool materials that would freeze when the temperature drops low enough. Once the liquid solidifies, radiation can no longer escape to space, so the cooling effect is cut off. And last October, Mandal and Yang reported another way to stop overcooling ${ }^{10}$. If they fill the pores of their polymer coating with isopropanol, the coating starts to trap heat rather than shed it. This can be reversed by blowing air through the pores to dry them out.

There's another issue: the materials achieve super-cooling only if they can send their radiation directly to the cold heat sink of outer space. In an urban setting, buildings, people and other objects can get in the way, absorbing the heat and re-emitting it. The best-performing materials currently remove heat at a rate of around $100 \mathrm{Wm}^{-2}$. Gan and Yu hope to double that by positioning their films perpendicular to the roof so that emissions can escape from both surfaces. But this will require adding materials around the films that can reflect the emissions up into the sky.

Researchers are looking at other ways to increase the materials' cooling ability. Last October, Evelyn Wang at the Massachusetts Institute of Technology in Cambridge and her colleagues reported that covering a radiative-cooling film with a light, insulating aerogel kept the structure $13^{\circ} \mathrm{C}$ cooler than its surroundings at noon in the dry Atacama Desert in Chile, compared with just $1.7^{\circ} \mathrm{C}$ without the aerogel ${ }^{3}$. The aerogel concept could be used with other super-cool materials, she says.
Dreams of using the super-cool materials for geoengineering to mitigate global warming seem further off, and unlikely from a practical perspective. Last September, Munday used "back-of-the-envelope calculations" to suggest that current rising temperatures could be balanced by covering 1-2\% of Earth's surface with existing materials that generate around $100 \mathrm{Wm}^{-2}$ of cooling power in the daytime ${ }^{11}$. But because solar panels still don't reach that level of cover after decades of development, it seems impossible that this nascent technology could do so in time to be useful, says Mark Lawrence, a climate scientist at the Institute for Advanced Sustainability Studies in Potsdam, Germany. As with any geoengineering proposal, Munday acknowledges the possible unintended consequences of disturbing precipitation patterns and local climates - which Ürge-Vorsatz agrees are likely to be a problem.

Still, passive radiative cooling might have many benefits, says Raman (see 'Electricity at night, water in the day'). It could, for instance help to stop solar panels losing efficiency as the temperature rises. And all electricity generation and conversion processes produce waste heat, says Yin, even if they use renewable energy rather than fossil fuels. "This is the only technology that harnesses all this wasted heat and dumps it back to space," he says.

XiaoZhi Lim is a freelance writer in Natick,

Massachusetts.

1. Raman, A. P., Anoma, M. A., Zhu, L., Rephaeli, E. \& Fan, S. Nature 515, 540-544 (2014).

2. Zhou, L. et al. Nature Sustain. 2, 718-724 (2019).

3. Leroy, A. et al. Sci. Adv. 5, eeat9480 (2019).

4. Rephaeli, E., Raman, A. \& Fan, S. Nano Lett. 13, 1457-1461 (2013).

5. Zhai, Y. et al. Science 355, 1062-1066 (2017).

6. Mandal, J. et al. Science 362, 315-319 (2018).

7. Li, T. et al. Science 364, 760-763 (2019).

8. Goldstein, E. A., Raman, A. P. \& Fan, S. Nature Energy 2, 17143 (2017).

9. Zhao, D. et al. Joule 3, 111-123 (2019).

10. Mandal, J. et al. Joule https://doi.org/10.1016/j. joule.2019.09.016 (2019)

11. Munday, J. N. Joule 3, 2057-2060 (2019).

12. Zhou, M. et al. Preprint at https://arxiv.org/abs/1804.10736 (2018).

13. Raman, A. P., Li, W. \& Fan, S. Joule 3, 2679-2686 (2019). 